\title{
Moment inequalities for the Boltzmann equation and applications to spatially homogeneous problems
}

\author{
A.V.Bobylev* \\ Department of Mathematics, \\ University of Kaiserslautern, \\ 67663 Kaiserslautern
}

\begin{abstract}
Some inequalities for the Boltzmann collision integral are proved. These inequalities can be considered as a generalization of the well-known Povmer inequality. The inequalities are used to obtain estimates of moments of solution to the spatially homogeneous Boltzmann equation for a wide class of intermolecular forces. We obtained simple necessary and sufficient conditions (on the potential) for the uniform boundedness of all moments. For potentials with compact support the following statement is proved: all moments of solution are bounded by corresponding moments of a certain Maxwellian $A \exp \left[-B(t) v^{2}\right]$ for any $t>0$, if this condition is fulfilled at $t=0$, moreover $B(t)=$ const for hard spheres. Estimate for a collision frequency are also obtained.
\end{abstract}

Key words: Boltzmann's equation, Povzner's inequality, moments.

\section{Introduction.}

The Boltzmann equation differs from other classical nonlinear equations of mathematical physics by its complexity. A five fold collision integral makes it very difficult to evaluate the right hand side of the equation even with a simple non-equilibrium distribution function.

The only exceptional case is the case of so-called Maxwell molecules, for which we can at least calculate in the closed form all moments of the collision integral. This simplification gives an opportunity to reduce the spatially homogeneous Boltzmann equation for Maxwell molecules to an infinite, but recurrently solvable, set of ODE for moments of the distribution function. This moment system of ODE makes it possible to apply different analytical methods and to study in detail properties of solutions [1].

However, the Maxwell model plays for the Boltamann equation (at least in the spatially. homogeneous case) almost the same role as equations with constant coefficients in the

*Permanent address: Keldysh Institute of Applied Mathematics, Ac of Sci of Russia, Miusskaya Sq.4, 125047 Moscow, Russia. 
general theory of PDE. Analytical methods become much less efficient if we try to apply them to other molecular models (for example, to hard spheres). At the same time one can make precise calculations with the Boltzmann equation by using modern computers. Therefore in the theory of this equation we need mostly qualitative results which can be expressed by inequalities. A simple idea to generalize some methods, which were previously used for Maxwellian molecules, is to derive from the Boltzmann equation a set. of differential inequalities for moments and then to study this set. Apparently the first step in this direction was made (in other terms) recently by Wennberg [2], who constructed a uniform upper bound for all moments of a spatially homogeneous solution in the case of hard potentials with angular cut-off (see also an important previous result of Desvillettes [3]). We use some ideas of [2] (the Povzner-type inequality and estimate of the loss term by Hölder's inequality) in the present paper.

The paper is organized as follows. In Sections 2-4 some useful inequalities for moments of the Boltzmann collision integral with arbitrary intermolecular potential are derived. To be more precise, in Section 2 we evaluate the integral of the Boltzmann collision operator with any convex function of energy and obtain a very simple estimate for hard spheres. In Section 2 we generalize this result to the case of arbitrary intermolecular forces. The main result of this part is formulated (Theorem 1) and discussed in Section 4. We note that these inequalities for the collision integral can be useful for different problems. However, we consider their applications only to the spatially homogeneous case. First, in Section 5, we generalize the above mentioned Wennberg's estimate to a very wide class of intermolecular forces including potentials with infinite radius of action. Simple necessary and sufficient conditions for uniform boundedness of moments are proved in this Section (Theorem 2). In Sections 6-7 we consider solutions with Maxwellian tails. The main results of these Sections are formulated in Theorem 3 (hard spheres) and Theorem 4 (potentials with compact support). Roughly speaking, we proved the following fact: if all moments of the initial condition are bounded by moments of the Maxwellian $A \exp \left(-B v^{2}\right)$, then all time-dependent moments of the solution are bounded by moments of the other Maxwellian $A_{1} \exp \left(-B_{1} v^{2}\right)$. This result is proved with $B_{1}=$ const for hard spheres and with $B_{1}=$ const $\cdot \mathrm{e}^{-\alpha \mathrm{t}}$ for an arbitrary potential with compact support.

\section{First inequality.}

Let $f(\mathrm{v}, t)$ be a time dependent distribution function in the velocity space, $\mathrm{v} \in R^{3}, t \geq$ 0 . We consider the spatially homogeneous Boltzmann equation

$$
\frac{\partial f}{\partial t}=I(f, f)=\int_{R^{3} \times S^{2}} d \mathbf{w} d \mathbf{n} u g\left(u, \frac{\mathrm{un}}{u}\right)\{f(\mathrm{v}) f(\mathbf{w})-f(\mathrm{v}) f(\mathrm{w})\}
$$

where

$$
\mathbf{u}=\mathbf{v}-\mathbf{w}, \quad u=|\mathbf{u}|, \quad|\mathbf{n}|=1, \quad \mathbf{v}=\frac{1}{2}(\mathbf{v}+\mathbf{w}+u \mathbf{n}), \quad \mathbf{w}=\frac{1}{2}(\mathbf{v}+\mathbf{w}-u \mathbf{n})
$$

The function $g(u, \cos \theta)$ is $a$ differential cross section, $0 \leq \theta \leq \pi$. In particular

$$
g(u, \cos \theta)=\frac{d^{2}}{4}=\text { const }
$$


for hard spheres with diameter $d$,

$$
g(u, \cos \theta)=u^{-4 / s} q_{s}(\cos \theta)
$$

for particles interacting with power-like potential $U(r)=$ const $\mathrm{r}^{-\mathrm{s}}, \mathrm{s}>1$.

For any isotropic test function $\psi\left(v^{2}\right)$ we put

$$
<\psi>=(f, \psi)=\int d \mathbf{v} f(\mathbf{v}, t) \psi\left(v^{2}\right)
$$

It follows from (1) that

$$
\frac{d}{d t}<\psi>=D(f \mid \psi)-\frac{1}{2} \int d \mathbf{v} d \mathbf{w} f(\mathbf{v}) f(\mathbf{w}) u A[\psi]
$$

where we omitted irrelevant argument $t$. The linear operator $A$ is defined by

$$
A[\psi]=\int_{S^{2}} d \mathbf{n} g\left(u, \frac{\mathrm{un}}{u}\right)\left[\psi\left(v^{2}\right)+\psi\left(w^{2}\right)-\psi\left(v^{2}\right)-\psi\left(w^{2}\right)\right]
$$

Our goal in this and next Sections is to establish some upper and lower bounds for $A[\psi]$. It is well known that a simple and very useful inequality for

$$
\Delta \psi=\psi\left(v^{2}\right)+\psi\left(w^{2}\right)-\psi\left(v^{2}\right)+\psi\left(w^{2}\right)
$$

with $\psi(z)=z^{p}$ was first obtained by Povzner [4]. One can find a derivation and applications of this inequality in the modern book [5]. Povzner's inequality was improved by Elmroth [6] and recently by Wennberg [2] who used it successfully to obtain some important estimates for moments of solutions of the Boltzmann equation (see Section 5 for details).

A simple idea of a further improvement of the Povzner-type inequalities is to estimate not the quantity (7), but its average $A[\psi](6)$. To do it let us consider an explicit form of the integral (6) in spherical coordinates with a polar axis directed along vector $\mathbf{u}=\mathbf{v}-\mathbf{w}$. We denote

$$
v=|\mathrm{v}|, \quad w=|\mathrm{w}|, \quad s=\sqrt{1-(\mathrm{vw} / v w)^{2}}, \mu=\frac{\mathrm{un}}{u}
$$

and notice that

$$
\begin{aligned}
& v^{2}=\frac{1}{2}\left(v^{2}+w^{2}+u|\mathrm{v}+\mathrm{w}| \cos \beta\right) \\
& w^{2}=\frac{1}{2}\left(v^{2}+w^{2}-u|\mathrm{v}+\mathrm{w}| \cos \beta\right) \\
& u^{2}|\mathrm{v}+\mathrm{w}|^{2}=\left(v^{2}+w^{2}\right)^{2}-4(\mathbf{v w})^{2}
\end{aligned}
$$

where $0 \leq \beta \leq \pi$ denotes the angle between vectors $\mathrm{n}$ and $(\mathrm{v}+\mathrm{w})$. In our coordinate system the integral (6) reads

$$
A[\psi]=\int_{-1}^{1} d \mu g(u, \mu) \int_{0}^{2 \pi} d \varphi\left[\psi\left(v^{2}\right)+\psi\left(w^{2}\right)-\psi\left(v^{2}\right)-\psi\left(w^{2}\right)\right],
$$


where $v^{2}$ and $w^{2}$ are defined by $(9)$ with

$$
\cos \beta=\mu \mu_{1}+\sqrt{1-\mu^{2}} \sqrt{1-\mu_{1}^{2}} \cos \varphi, \quad \mu_{1}-\frac{\mathrm{u}(\mathrm{v}+\mathrm{w})}{u|\mathrm{v}+\mathrm{w}|}
$$

Noting that

$$
\mathbf{u}(\mathbf{v}+\mathbf{w})=v^{2}-w^{2}, \quad u|\mathbf{v}+\mathbf{w}| \cos \beta=\mu\left(v^{2}-w^{2}\right)+2 v w \sqrt{1-\mu^{2}} s \cos \varphi,
$$

we obtain from $(9)$

$$
\begin{gathered}
v^{2}=\frac{v^{2}+w^{2}}{2}\left(1+\mu \mu_{0}+\sqrt{1-\mu^{2}} \sqrt{1-\mu_{0}^{2}} s \cos \varphi\right), \quad v^{2}=\frac{v^{2}+w^{2}}{2}\left(1+\mu_{0}\right), \\
w^{2}=\frac{v^{2}+w^{2}}{2}\left(1-\mu \mu_{0}-\sqrt{1-\mu^{2}} \sqrt{1-\mu_{0}^{2}} s \cos \varphi\right), \quad w^{2}=\frac{v^{2}+w^{2}}{2}\left(1-\mu_{0}\right) \\
\mu_{0}=\frac{v^{2}-w^{2}}{v^{2}+w^{2}}
\end{gathered}
$$

Formulas (10)-(11) give an explicit representation of the integral $A[\psi]$. Now one can easily prove the following estimates.

Lemma 2.1. If $\psi(z)$ is a convex function, then

$$
A_{-}[\psi] \leq A[\psi] \leq A_{+}[\psi]
$$

where

$$
\begin{gathered}
\left.A_{-}[\psi]=\int_{0}^{1} d x b(u, x)\left\{\psi\left[x v^{2}+(1-x) w^{2}\right]+\psi\left[x w^{2}+(1-x) v^{2}\right]-\psi\left(v^{2}\right)-\psi, w^{2}\right)\right\} \\
b(u, x)=4 \pi g(u, 1-2 x), \quad u=|\mathrm{v}-\mathrm{w}| \\
A_{+}[\psi]=\int_{-1}^{1} d \mu\left\{\psi\left[\frac{v^{2}+w^{2}}{2}(1+\mu)\right]+\psi\left[\frac{v^{2}+w^{2}}{2}(1-\mu)\right]-\psi\left(v^{2}\right)-\psi\left(w^{2}\right)\right\} \int_{0}^{2 \pi} d \varphi g(u, \cos \gamma), \\
\cos \gamma=\mu \mu_{0}+\sqrt{1-\mu^{2}} \sqrt{1-\mu_{0}^{2}} \cos \varphi, \quad \mu_{0}=\frac{v^{2}-w^{2}}{v^{2}+v^{2}} .
\end{gathered}
$$

Proof. We put in (11)

$$
\begin{gathered}
v^{2}=B_{+}+C s \cos \varphi, \quad w^{2}=B_{-}-C s \cos \varphi, \quad B_{ \pm}=\frac{v^{2}+w^{2}}{2}\left(1 \pm \mu \mu_{0}\right), \\
C=\frac{v^{2}+w^{2}}{2} \sqrt{1-\mu^{2}} \sqrt{1-\mu_{0}^{2}} s=\sqrt{1-(\mathrm{vw} / v w)^{2}}
\end{gathered}
$$

and consider the inner integral in (9)

$$
K(s)=\int_{0}^{2 \pi} d \varphi\left[\psi\left(v^{2}\right)+\psi\left(w^{2}\right)-\psi\left(v^{2}\right)-\psi\left(w^{2}\right)\right]
$$


as a function of $0 \leq s \leq 1$. For a proof of (12)-(14) it is sufficient to prove that $K(0)<$ $K(s) \leq K(1)$ for any convex function $\psi(z)$. First we consider the integral

$$
K_{1}(s)=\int_{0}^{2 \pi} d \varphi\left[\psi\left(v^{2}\right)-\psi\left(v^{2}\right)\right]=\int_{0}^{2 \pi} d \varphi\left[\psi\left(B_{+}+C s \cos \varphi\right)-\psi\left(v^{2}\right)\right] .
$$

An elementary transformation gives

$$
K_{1}(s)=2 \int_{0}^{1} \frac{d \tau}{\sqrt{1-\tau^{2}}}\left[\psi\left(B_{+}+C s \tau\right)+\psi\left(B_{+}-C s \tau\right)-2 \psi\left(v^{2}\right)\right] .
$$

For smooth function $\psi(z)$ we obtain

$$
\frac{\partial K}{\partial s}=2 C \int_{0}^{1} \frac{\tau d \tau}{\sqrt{1-\tau^{2}}}\left[\psi^{\prime}\left(B_{+}+C s \tau\right)-\psi^{\prime}\left(B_{+}-C s \tau\right)\right] \geq 0
$$

since $C>0$ and $\psi^{\prime \prime}(z) \geq 0$ for any convex function. For the general case we can use the fact that the convex function $\psi(z)$ has almost every where a monotonically nondecreasing derivative $\psi(z)$ (see, for example, [7]). Therefore the equality (15) proves that $K_{1}(0) \leq K_{1}(s) \leq K(1)$. Repeating the same considerations for

$$
K_{2}(s)=\int_{0}^{2 \pi} d \varphi\left[\psi\left(w^{2}\right)-\psi\left(w^{2}\right)\right]
$$

one can conclude that

$$
\begin{gathered}
K(0) \leq K(s)=K_{1}(s)+K_{2}(s) \leq K(1), \\
K(0)=2 \pi\left\{\psi\left[\frac{v^{2}+w^{2}}{2}\left(1+\mu \mu_{0}\right)\right]+\psi\left[\frac{v^{2}+w^{2}}{2}\left(1-\mu \mu_{0}\right)\right]-\psi\left(v^{2}\right)-\psi\left(w^{2}\right)\right\}, \\
K(1)=\int_{0}^{2 \pi} d \varphi\left\{\psi\left[\frac{v^{2}+v^{2}}{2}(1+\cos \gamma)\right]+\psi\left[\frac{v^{2}+w^{2}}{2}(1-\cos \gamma)\right]-\psi\left(v^{2}\right)-\psi\left(w^{2}\right)\right\},
\end{gathered}
$$

$\mu_{0}$ and $\cos \gamma$ being defined in (14). Substituting the innet integral in (10) by $K(0)$ and changing variables to $x=(1-\mu) / 2$, we just obtain the lower estimate (13). Then we substitute $K(1)$ into $(10)$ and use a symmetry property

$$
\int_{-1}^{1} d \mu \int_{0}^{2 \pi} d \varphi F_{1}(\mu) F_{2}(\cos \gamma)=\int_{-1}^{1} d \mu \int_{0}^{2 \pi} d \varphi F_{2}(\mu) F_{1}(\cos \gamma)
$$

which is obviously valid for any $-1 \leq \mu_{0} \leq 1$ in $(14)$. It results in the upper estimate (14) and completes the proof.

As an example of estimates (12)-(14) we consider the case of hard spheres (2). Then

$$
A-[\psi]=\pi d^{2}\left[\frac{2}{v^{2}-w^{2}} \int_{w^{2}}^{2} d z \psi(z)-v\left(v^{2}\right)-\psi\left(w^{2}\right)\right]
$$




$$
A_{+}[\psi]=\pi d^{2}\left[\frac{2}{v^{2}+w^{2}} \int_{0}^{v^{2}+w^{2}} d z \psi(z)-\psi\left(v^{2}\right)-\psi\left(w^{2}\right)\right] .
$$

If $\psi(z)=z^{n}, n=2,3, \ldots$, then we obtain the folluwing simple inequality

$$
\frac{2 \pi d^{2}}{n+1} \sum_{k=1}^{n-1} v^{2 k} w^{2(n-k)} \leq A\left[v^{2 n}\right]+\frac{n-1}{n+1}\left(v^{2 n}+w^{2 n}\right) \leq \frac{2 \pi d^{2}}{n+1} \sum_{k=1}^{n-1}\left(\begin{array}{l}
n \\
k
\end{array}\right) v^{2 k} w^{2(n-k)},
$$

which demonstrates an accuracy of our estimates.

Thus inequalities (12) (14) are sufficiently simple and convenicnt for the case of isotropic scattering, i.e. for $g(u, \cos \theta)=\tilde{g}(u)$. The most important upper bound $A_{+}[\psi](14)$ is still too complicated in general case. Therefore it is desirable to simplify the integral (14) and to obtain approximate formulas like (16) for the case of non-isotropic scattering. We consider this problem in Section 3.

\section{Second inequality.}

To study the integral (14) one can fix $\mathrm{v}$ and $\mathrm{w}$ and simplify notations omitting all irrelevant arguments $v^{2}, w^{2}, u=|\mathbf{v}-\mathbf{w}|$. Then we rewrite (14) as a function of $\mu_{0}=$ $\left(v^{2}-w^{2}\right) /\left(v^{2}+w^{2}\right)$

$$
\begin{gathered}
A_{+}[\psi]=F\left(\mu_{0}\right)=\int_{-1}^{1} d \mu \int_{0}^{2 \pi} d \varphi g(\cos \gamma)\left[\Phi(\mu)-\Phi\left(\mu_{0}\right)\right] \\
\Phi(\mu)=\psi\left[\frac{v^{2}+w^{2}}{2}(1+\mu)\right]+\psi\left[\frac{v^{2}+w^{2}}{2}(1-\mu)\right], \\
\cos \gamma=\mu \mu_{0}+\sqrt{1-\mu^{2}} \sqrt{1-\mu_{0}^{2}} \cos \varphi, \quad \tilde{g}(\cos \gamma)=g(u, \cos \gamma),
\end{gathered}
$$

tilda being omitted below.

The integral (18) is nothing but the one speed collision operator, its properties are well - known from the linear transport theory [8]. In particular, for

$$
\Phi(\mu)=\sum_{n=0}^{\infty} a_{n} P_{2 n}(\mu), \quad a_{n}=\frac{2}{4 n+1} \int_{-1}^{1} d \mu \Phi(\mu) P_{2 n}(\mu)
$$

we obtain

$$
F\left(\mu_{0}\right)=-\sum_{n=1}^{\infty} g_{n} a_{n} P_{2 n}\left(\mu_{0}\right), \quad g_{n}=2 \pi \int_{-1}^{1} d_{\mu l}(\mu)\left[1-P_{2 n}(\mu)\right]
$$

where $P_{2 n}(\mu)$ are Legendre's polynomials (note that $\Phi(\mu)$ in (18) is always even function).

Remark. To prove (21) it is sufficient to substitute (20) into (18) and use the addition theorem for $P_{2 n}(\mu)$.

If $g=$ const, then (18) can be written as

$$
F\left(\mu_{0}\right)=F(1)+\sigma\left[\Phi(1)-\Phi\left(\mu_{0}\right)\right], \quad \sigma=4 \pi g=\text { const. }
$$


This formula is convenient for an even convex function $\Phi(\mu)$ since $\Phi(0) \leq \Phi(1)$ and $F(1) \leq 0$. Let us try to estimate the difference $\left[F\left(\mu_{0}\right)-F(1)\right]$ in the general case. Using the expansion (20) we obtain

$$
F\left(\mu_{0}\right)-F(1)=\sum_{n=1}^{\infty} g_{n} a_{n}\left[1-P_{2 n}\left(\mu_{0}\right)\right]
$$

Suppose that $a_{n} \geq 0$ for all $n=1,2, \ldots$ and

$$
\sigma=2 \pi \int_{-1}^{1} \operatorname{dng}(\mu)<\infty
$$

then all terms in $(23)$ are non-negative $\left(\left|P_{n}(\mu)\right| \leq 1\right)$ and

$$
F\left(\mu_{0}\right)-F(1) \leq\|g\|\left[\Phi(1)-\Phi\left(\mu_{0}\right)\right], \quad\|g\|=\sup _{n \geq 1} g_{n} \leq 2 \sigma
$$

The condition (24) is not valid for long range potentials. However in this case one can easily estimate (23) for a polynomial case

$$
\Phi(\mu)=\sum_{n=0}^{N} a_{n} P_{2 n}(\mu), \quad a_{n} \geq 0 .
$$

Then

$$
F\left(\mu_{0}\right)-F(1) \leq\left\|g_{N}\right\|\left[\Phi(1)-\Phi\left(\mu_{0}\right)\right], \quad\left\|g_{N}\right\|=\max _{1 \leq n \leq N} g_{n}
$$

Thus we do obtain simple expressions (25), (26) for upper bounds of (19) provided that all coefficients $a_{n}$ in (20) are non negative. Let us check this condition for the most important case $\psi(z)=z^{p}(p>1)$ in (18). Then $([9], p .837)$

$$
\begin{array}{r}
\Phi(\mu)=\left(v^{2}+w^{2}\right)^{p}\left[\left(\frac{1+\mu}{2}\right)^{p}+\left(\frac{1-\mu}{2}\right)^{p}\right], \\
a_{n}=\frac{16\left(v^{2}+w^{2}\right)^{p}}{4 n+1} \int_{0}^{1} d x^{2 p+1} P_{2 n}\left(1-2 x^{2}\right)=\frac{8\left(v^{2}+w^{2}\right)^{p}[\Gamma(p+1)]^{2}}{(4 n+1) \Gamma(p+2 n+2) \Gamma(p+1-2 n)}
\end{array}
$$

so that a sign of $a_{n}$ is defined by

$$
\frac{\Gamma(p+1)}{\Gamma(p+1-2 n)}-\prod_{k=0}^{2 n-1}(p-k)-\left\{\prod_{k=0}^{[p]}(p-k)\right\}(-1)^{[p]+1} \prod_{k=[p]+1}^{2 n-1}(k-p)
$$

where $[p]$ denotes an integer part of $p$ (the second formula makes sense only for $p<2 n-1$ ). Hence, the condition $a_{n} \geq 0$ is fulfilled for $\psi=z^{p}$ if (1) $p=2,3, \ldots$ is an integer number or (2) $2 m+1<p<2 m+2$ for certain integer $m \geq 0$. The coefficients $a_{n}$ are negative if $2 m<p<2 m+1$ and $2 n>p+1$.

If coefficients $a_{n}$ are partly negative, then we put

$$
\Phi(\mu)=\Phi_{+}(\mu)-\Phi-(\mu)=\Sigma a_{n} P_{2 n}(\mu)-\Sigma\left|a_{n}\right| P_{2 n}(\mu),
$$


where the $\operatorname{sum} \Sigma^{\prime}\left(\Sigma^{\prime \prime}\right)$ is taken over such $n \geq 1$, that $a_{n}>0\left(a_{n}<0\right)$. We also assume that $\sigma<\infty$ and

$$
1 \leq \lambda=\sup _{-1 \leq \mu \leq 1} \frac{\Phi_{+}(1)-\Phi_{+}(\mu)}{\Phi(1)-\Phi(\mu)}<\infty, \quad \Phi(\mu) \leq \Phi(1),
$$

then the sum (23) can be estimated by

$$
\begin{array}{r}
F\left(\mu_{0}\right)-F(1) \leq \sigma \sum_{n=1}^{\infty} a_{n}\left[1-P_{2 n}\left(\mu_{0}\right)\right]+\|\hat{g}\| \sum_{n=1}^{\infty}\left|a_{n}\right|\left[1-P_{2 n}\left(\mu_{0}\right)\right] \\
\|\hat{g}\|=\sup _{n \geq 1}\left|2 \pi \int_{-1}^{1} d \mu g(\mu) P_{2 n}(\mu)\right|
\end{array}
$$

Noting that

$$
\begin{gathered}
\sum_{n=1}^{\infty} a_{n}\left[1-P_{2 n}(\mu)\right]=\Phi(1)-\Phi(\mu), \\
\sum_{n=1}^{\infty}\left|a_{n}\right|\left[1-P_{2 n}(\mu)\right]=2\left[\Phi_{+}(1)-\Phi_{+}(\mu)\right]-[\Phi(1)-\Phi(\mu)] \leq(2 \lambda-1)[\Phi(1)-\Phi(\mu)]
\end{gathered}
$$

we obtain

$$
F\left(\mu_{0}\right)-F(1) \leq[\sigma+(2 \lambda-1)\|\hat{g}\|]\left[\Phi(1)-\Phi\left(\mu_{0}\right)\right]
$$

Collecting some inequalities of this Section we can formulate the following lemma.

Lemma 3.1. Let the function $F\left(\mu_{0}\right)$ be defined by $(18), g(\mu) \geq 0$.

[i] If

$$
\Phi(\mu)=\sum_{n=0}^{N} a_{n} P_{2 n}(\mu), \quad a_{n} \geq 0 ; \quad \int_{-1}^{1} d \mu g(\mu)\left(1-\mu^{2}\right)<\infty
$$

then

$$
F\left(\mu_{0}\right) \leq F(1)+\left\|g_{N}\right\|\left[\Phi(1)-\Phi\left(\mu_{0}\right)\right], \quad\left\|g_{N}\right\|=\max _{1 \leq n \leq N}\left\{2 \pi \int_{-1}^{1} d \mu g(\mu)\left[1-P_{2 n}(\mu)\right]\right\}
$$

[ii] If

$$
\Phi(\mu)=\sum_{n=0}^{\infty} a_{n} P_{2 n}(\mu), \quad \sum_{n=0}^{\infty}\left|a_{n}\right|<\infty, \quad \sigma=2 \pi \int_{-1}^{1} d \mu g(\mu)<\infty,
$$

and the conditions (29) are fulfilled, then

$$
F\left(\mu_{0}\right) \leq F(1)+\left[\sigma+(2 \lambda-1) \| \hat{g} \mid[]\left[\Phi(1)-\Phi\left(\mu_{0}\right)\right]\right.
$$

where $\lambda$ is defined by (29),

$$
\|\hat{g}\|=\sup _{n \geq 1}\left|2 \pi \int_{-1}^{1} d \mu g(\mu) P_{2 n}(\mu)\right| \leq \sigma .
$$




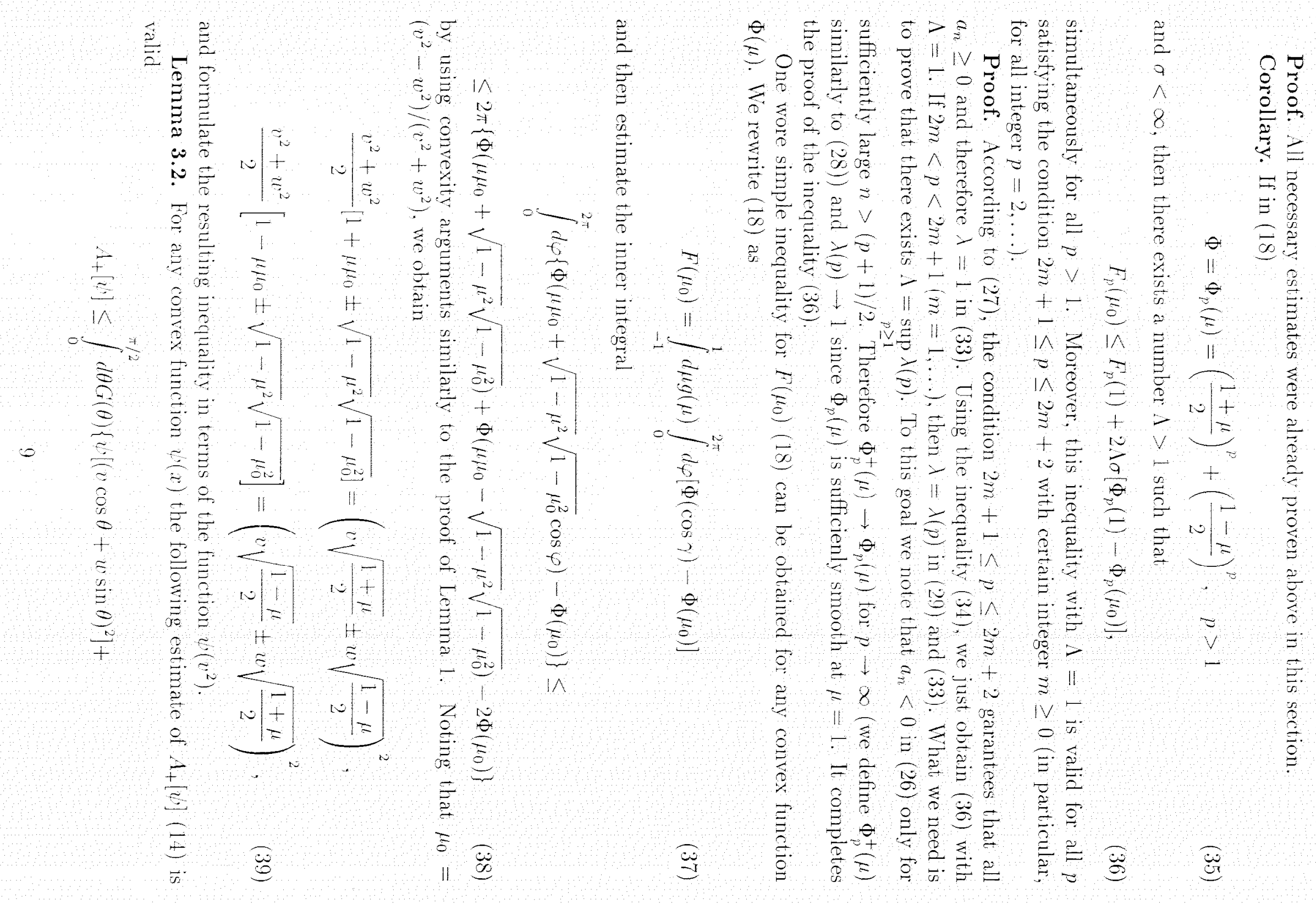




$$
\left.+\psi\left[(w \cos \theta-v \sin \theta)^{2}\right]-\psi\left(v^{2}\right)-\psi\left(w^{2}\right)\right\}
$$

where

$$
G(\theta)=2 \pi \sin 2 \theta[g(u, \cos 2 \theta)+g(u,-\cos 2 \theta)] .
$$

Proof. We estimate (37) by the inequality (38), then substitute $\Phi(\mu)$ by $\psi(z)(19)$ and use formulas (39). Putting $\mu=\cos 2 \theta$ in (37) we obtain the final result (40).

Remark. This lemma shows that one can use the simple Kac equation to estimate integrals (5) for a convex function $\psi(z)$. Moreover it is possible to use (40) for a simplified proof of Lemma 3.1 (with a convex function $\Phi(\mu)$ !) changing $P_{2 n}(\cos \theta)$ to $\cos 2 n \theta$. However we prefer more general result without using a convexity.

\section{Estimates for moments of the collision term.}

It is convenient to put together some relevant estimates from Sections 2-3 and formulate the result in terms of equalities (4)-(6). We assume that $A[\psi]$ is defined by the cquality $(6), g(u, \mu) \geq 0$ and

$$
\sigma^{(2)}(u)=2 \pi \int_{-1}^{1} d \mu g(u, \mu)\left(1-\mu^{2}\right)<\infty
$$

We also assume that $\psi(z)$ has a continuous derivative $\psi(z), z \geq 0$, to garantee a convergence of the integral (6) for long range potentials. Then the following theorem is valid.

Theorem 1. [i] If $\psi(z)$ is a convex function, then

$$
\begin{gathered}
A[\psi] \geq \int_{0}^{1} d x b(u, x)\left\{\psi\left[x v^{2}+(1-x) w^{2}\right]+\psi\left[x w^{2}+(1-x) v^{2}\right]-\psi\left(v^{2}\right)-\psi\left(w^{2}\right)\right\} \\
b(u, x)=2 \pi g(u, 1-2 x), \quad u=|\mathrm{v}-\mathrm{w}| \\
A[\psi] \leq \int_{0}^{\pi / 2} d \theta G(u, \theta)\left\{\psi\left[(v \cos \theta+w \sin \theta)^{2}\right]+\psi\left[(v \sin \theta-w \cos \theta)^{2}\right]-\psi\left(v^{2}\right)-\psi\left(w^{2}\right)\right\} \\
G(u, \theta)=2 \pi \sin 2 \theta[g(u, \cos 2 \theta)+g(u,-\cos 2 \theta)]
\end{gathered}
$$

[ii] If $\psi(z)$ is a convex function and

$$
\int_{-1}^{1} d \mu \psi\left[z \frac{1+\mu}{2}\right] P_{2 n}(\mu) \geq 0, n=1, \ldots, \quad z>0
$$

then

$$
\begin{gathered}
A[\psi] \leq g_{*}\left[\psi\left(v^{2}+w^{2}\right)+\psi(0)-\psi\left(v^{2}\right)-\psi\left(w^{2}\right)\right]- \\
-2 \pi \int_{-1}^{1} d \mu g(u, \mu)\left\{\psi\left(v^{2}+w^{2}\right)+\psi(0)-\psi\left[\frac{v^{2}+w^{2}}{2}(1+\mu)\right]-\psi\left[\frac{v^{2}+w^{2}}{2}(1-\mu)\right]\right\},
\end{gathered}
$$




$$
g_{*}=g_{*}(u)=\sup \left\{g_{n}=2 \pi \int_{-1}^{1} d \mu g(u, \mu)\left[1-P_{2 n}(\mu)\right] \mid n-1, \ldots ; a_{n}>0\right\} .
$$

[iii] If $\psi(z)=z^{p}$ and

$$
\sigma=2 \pi \int_{-1}^{1} d \mu g(u, \mu)<\infty
$$

then there exists such number $\Lambda \geq 1$ that for all $p \geq 1$

$$
\begin{aligned}
& A\left[v^{2 p}\right]<-\lambda_{p}\left(v^{2}+w^{2}\right)^{p}+2 \Lambda \sigma\left[\left(v^{2}+w^{2}\right)^{p}-v^{2 p}-w^{2 p}\right] \\
& \lambda_{p}=\lambda_{p}(u)=2 \pi \int_{-1}^{1} d \mu g(u, \mu)\left[1-\left(\frac{1+\mu}{2}\right)^{p}-\left(\frac{1 \mu}{2}\right)^{p}\right]
\end{aligned}
$$

this inequality with $A=1$ being valid for all $p$ such that $2 m-1 \leq p \leq 2 m$ for a certain integer number $m=1,2, \ldots$.

Proof. Inequalities (42) and (43) are taken from Lemma 2.1 and Lemma 3.2. The inequality (45) was proven in Lemma 3.1. Moment estimates (47) can be easily obtained by substitution $\psi(z)=z^{p}$ into (18)-(19) and using the inequality (36). It completes a proof of the theorem.

Remark. If $g(u, \mu)$ doos not depend on $\mu$ (isotropic scattering), then (47) is valid even with $\Lambda=1 / 2$. In this special case it is more convenient to use directly Lemma 2.1 with simple formulas (16), where $d=d(u)$ for "soft spheres".

In order to apply the theorem to the Boltzmann equation (1) with long range potentials we consider $(45)$ with $\psi(z)=z^{m}, m=1,2, \ldots$ Then

$$
A\left[z^{m}\right] \leq g_{*}\left[\left(v^{2}+w^{2}\right)^{m}-v^{2 m}-w^{2 m}\right]-\lambda_{m}\left(v^{2}+w^{2}\right)^{m}
$$

where $\lambda_{m}$ is defined by (48),

$$
g_{*}=\max _{1 \leq n \leq m}\left\{2 \pi \int_{-1}^{1} \operatorname{d\mu g}(u, \mu)\left[1-P_{2 n}(\mu)\right]\right\}
$$

Putting

$$
B_{n}-\max _{1 \leq n \leq m}\left\{\sup _{0 \leq \mu \leq 1} \frac{1-P_{2 n}(\mu)}{1-\mu^{2}}\right\}
$$

we obviously obtain $g_{*}(u) \leq B_{m} \sigma^{(2)}(u)(41)$

Moreover:

$$
\lambda_{p}(u) \geq \lambda_{2}(u)=\frac{1}{2} \sigma^{(2)}(u), \quad p \geq 2
$$

since $\partial \lambda_{p} / \partial p>0$. Therefore we obtain from (49) a simple estimate

$$
A\left[v^{2 m}\right] \leq \frac{1}{2} \sigma^{(2)}(u)\left\{2 B_{m}\left[\left(v^{2}+w^{2}\right)^{m}-v^{2 m}-v^{2 m}\right]-\left(v^{2}+w^{2}\right)^{m}\right\}, \quad m=2,3, \ldots,
$$

where $B_{m}(50)$ depends only on number $m$. It is clear that $B_{m} \geq B_{2}=3 / 2$. 
On the other hand, it follows from (42) that

$$
A\left[v^{2 m}\right] \geq \sum_{k=1}^{m-1}\left(\begin{array}{c}
m \\
k
\end{array}\right)\left[v^{2 k} w^{2(m-k)}+w^{2 k} v^{2(m-k)}-v^{2 m}-w^{2 m}\right] \int_{0}^{1} d x g(u, x) x^{k}(1-x)^{m-k}
$$

Let $v \geq w$, then

$$
\begin{gathered}
A\left[v^{2 m}\right] \geq-v^{2 m} \sum_{k=1}^{m-1}\left(\begin{array}{c}
m \\
k
\end{array}\right)\left[1-\left(\frac{w}{v}\right)^{2 k}\right]\left[1-\left(\frac{w}{v}\right)^{2(m-k)}\right] \int_{0}^{1} d x b(u, x) x^{k}(1-x)^{m-k} \geq \\
\geq-v^{2 m} \int_{0}^{1} d x b(u, x)\left[1-x^{m}-(1-x)^{m}\right]
\end{gathered}
$$

Using the elementary inequality

$$
1-x^{m}-(1-x)^{m}=x(1-x) \sum_{k=0}^{m-2}\left[x^{k}+(1-x)^{k}\right] \leq 2(m-1) x(1-x)
$$

and a definition $(42)$ of $b(u, x)$, we obtain a lower bound

$$
A\left[v^{2 m}\right] \geq-\frac{m-1}{2} \sigma^{(2)}(u) \max \left(v^{2 m}, w^{2 m}\right), \quad m=1, \ldots
$$

Using (52), (53) one can easily prove the following statement.

Corollary 1. Moments (5) of the Boltzmann collision integral (1) satisfy inequalities

$$
\begin{gathered}
D\left(f \mid v^{2 n}\right) \geq-\frac{n-1}{2} \int d \mathbf{v} d \mathbf{w} f(\mathbf{v}) f(\mathbf{w}) u \sigma^{(2)}(u) v^{2 n} \\
D\left(f \mid v^{2 n}\right) \leq-\left(2^{n-1}-1\right)\left(2 B_{n}-1\right) \int d \mathbf{v} d \mathbf{w} f(\mathbf{v}) f(\mathbf{w}) u \sigma^{(2)}(u) w v^{2 n-1}- \\
-\frac{1}{2} \int d \mathbf{v} d \mathbf{w} f(\mathbf{v}) f(\mathbf{w}) u \sigma^{(2)}(u) v^{2 n}, \quad u=|\mathbf{v}-\mathbf{w}|, \quad n=2,3, \ldots,
\end{gathered}
$$

where $\sigma^{(2)}(u)$ and $B_{n}$ are defined in $(41)$ and $(50)$.

Proof. It is sufficient to substitute (52) and (53) into (5) and use elementary inequalities

$$
\begin{gathered}
\max (x, y) \leq x+y, \quad(x+y)^{n}-x^{n}-y^{n}=\sum_{k=1}^{n-1}\left(\begin{array}{l}
n \\
k
\end{array}\right) x^{k} y^{n-k} \leq \\
\leq 2\left(2^{n-1}-1\right)\left(x^{1 / 2} y^{n-1 / 2}+y^{1 / 2} x^{n-1 / 2}\right), \quad x \geq 0, \quad y \geq 0, \quad n=2,3, \ldots
\end{gathered}
$$

Estimates (54), (55) are especially convenient for longe range potential since the crosssection $\sigma^{(2)}(u)$ is always finite (otherwise the integral over unit sphere (6) diverges even for differentiable function $\psi(z)$ ). However these estimates can be improved in the case of potentials with finite radius of action. In this case a total cross section (46) is finite and we can apply the inequality (47) and obtain the following result. 
Corollary 2. If the condition (46) is fulfilled, then for any $p_{0}>1$ and for all $p \geq p_{0}$

$$
\begin{aligned}
& D\left(f \mid v^{2 p}\right) \leq \Lambda \sigma \int d \mathbf{v} d \mathbf{w} f(v) f(\mathbf{w}) u\left[\left(v^{2}+w^{2}\right)^{p}-v^{2 p}-w^{2 p}\right]- \\
& -\frac{1}{2} \int d \mathbf{v} d \mathbf{w} f(\mathbf{v}) f(\mathbf{w}) u \lambda_{p o}(u)\left(v^{2}+w^{2}\right)^{p}, \quad u=|\mathbf{v}-\mathbf{w}|
\end{aligned}
$$

where $\Lambda \geq 1$ and $\lambda_{p_{0}}(u) \leq \sigma$ are defined in Theorem 1 [iii].

Proof. We substitute (47) into (5) and use the fact that $\lambda_{p}(u)(48)$ is a monotone function of $p \geq 1$.

Inequalities (54)-(56) will be used below to estimate moments of the distribution function $f(\mathrm{v}, t)$.

\section{Moments of the solution.}

We consider the Boltzmann equation (1) with initial condition

$$
f_{\left.\right|_{t=0}}-f_{0}(\mathbf{v}), \quad \int d \mathbf{v} f_{0}(\mathbf{v})-1, \int d \mathbf{v} v^{2} f_{0}(\mathbf{v})=E_{0}, \quad H_{0}-\int d \mathbf{v} f_{0}(\mathbf{v}) \ln f_{0}(\mathbf{v})
$$

and assume that there exists a solution $f(\mathrm{v}, t)$ of the Cauchy problem (1), satistying conservation laws and H-theorem (see [5] for a review of existence theorems)

$$
\int d \mathbf{v} f(\mathbf{v}, t)=1, \quad \int d \mathbf{v} f(\mathbf{v}, t) v^{2}=F_{0}, \quad \int d \mathbf{v} f(\mathbf{v}, t) \ln f(\mathbf{v}, t) \leq H_{0}
$$

Moreover we assume formally that all integer moments of the solution

$$
m_{n}(t)=\int d \mathbf{v} f(\mathbf{v}, t) v^{2 n}, \quad n=1,2, \ldots
$$

are finite. Our aim is to estimate upper and lower bounds of $m_{n}(t)$. This problem was recently considered by Wennberg [2] (see also the previons paper of Desvilletes [3]) who obtained such estimates for hard splieres and power-line potentials with angular cut-off. Wennberg's results are based on his version of the Povzner inequality for $\Delta \psi(z)$. Using the stronger inequality (55) and following the same ideas one can easily generalize the results of [2] to a very wide class of intermolecular potentials. to be more precise we assume that:

$$
c_{1} u \leq u \sigma^{(2)}(u) \leq c_{2} u+c_{3}
$$

for certain $c_{1.2,3}>0$ and $0<\gamma \leq 1$.

Theorem 2. If $\sigma^{(2)}(u)(41)$ satisfies the condition $(60)$, then all moments (59) of the solution $f(v, t)$ of the Cauchy problem (1), (57) satisfy mequalities

$$
m_{n}(t) \leq m_{n}^{*}\left[1-e^{-\lambda_{n} t}\right]^{-\gamma / 2 n}, n=2,3 \ldots
$$

where the constants $m_{n}^{*}, \lambda_{n}$ depend only on $n, E_{0}, H_{0}, \gamma, c_{1,2,3}$. If $u \sigma^{(2)}(u) \leq c_{3}$, then

$$
m_{n}(t) \geq m_{n}(0) \exp \left[-\frac{n-1}{2} c_{3} t\right], n=1, \ldots
$$


Remark. Inequalities similar to (61) were first obtained by Wennberg [3] for a special case

$$
g(u, \mu)=u^{\beta} h(\mu), \quad \int_{-1}^{1} d \mu h(\mu), \infty, \quad 0 \leq \beta<1 .
$$

He also noted that they are not valid for pseudo-Maxwell molecules $(\beta-1)$.

Proof. Noting that (5)

$$
\frac{d m_{n}(t)}{d t}-D\left(f \mid v^{2 n}\right)
$$

and using the lower estimate (54) we immediately obtain the second inequality (62). To prove (61) we merely repeat Wennberg's arguments [2]. First we apply the Arkeryd result [10]

$$
\int d \mathbf{w} f(\mathbf{w}, t)|\mathbf{v}-\mathbf{w}|^{\gamma} \geq q\left(\gamma, E_{0}, H_{0}\right) v^{\gamma}
$$

to a negative term in $(55)$ and obtain

$$
\int d \mathbf{v} d \mathbf{w} f(\mathbf{v}, t) f(\mathbf{w}, t) u \sigma^{(2)}(u) w^{2 n} \geq c_{1} q \int d \mathbf{v} f(\mathbf{v}, t) v^{2 n+\gamma}
$$

Then we note that

$$
\int d \mathbf{v} f(\mathbf{v}, t) v^{2 n+\gamma} \geq\left[m_{n}(t)\right]^{1+\gamma / 2 r_{b}}
$$

because of the Hölder inequality and the normalization condition $m_{0}(t)=1(58)$.

To estimate a positive term in (55) we note that $w<1+w^{2}, v^{2 n-1}<1+v^{2 n}$ and $u \leq 1+v+w$. Therefore

$$
\int d \mathbf{v} d \mathbf{w} f(\mathbf{v}, t) f(\mathbf{w}, t)\left[c_{2} u+c_{3}\right] w v^{2 n-1} \leq\left(c_{3}+3 c_{2}\right)\left(1+E_{0}\right)\left[1+m_{n}(t)\right]
$$

Substituting (65)-(67) into (55) and (63) we obtain a differential inequality

$$
\frac{d m_{n}}{d t} \leq A_{n}\left(1+m_{n}\right)-D m_{n}^{1+\gamma / 2 n}, \quad n=2, \ldots
$$

with

$$
A_{n}=\left(2^{n-1}-1\right)\left(2 B_{n}-1\right)\left(c_{3}+3 c_{2}\right)\left(1+E_{0}\right), \quad D=\frac{1}{2} c_{1} q
$$

A substitution

$$
m_{n}(t)=\left[y\left(\frac{2 n}{\gamma} D t\right)\right]^{-2 n / \gamma}
$$

results in the incquality

$$
\frac{d y}{d t}+a y\left[1+y^{b}\right] \geq 1, \quad b=\frac{2 n}{\gamma}, \quad a=\frac{\gamma}{2 n} \frac{A_{n}}{D}
$$

One can easily prove (see Lemma 6.3 in Section 6 for details) that

$$
y(t) \geq y_{*}\left(1-e^{-a t}\right)
$$


where $y_{*}$ is a unique positive root of algebraic equation $a y_{*}\left(1+y_{*}^{b}\right)=1$. Hence, the moments (65) satisfy inequalities (61) with $\lambda_{n}=A_{n}, m_{n}^{*}=\left(y_{*}\right)^{-2 n / \gamma}$. It completes the proof:

Conditions (60) has a clear physical meaning. If $\sigma^{(2)}(u)(41)$ is finite, then the upper bound in (60) is almost trivial. This condition is fulfilled for any physically relevant intermolecular potential $U(r)$, where $r>0$ denotes a distance between two particles. Under some natural restrictions on the potential $U(r)$ the lower bound in $(60)$ is equivalent to an asymptotic inequality

$$
\sigma^{(2)}(u) \geq c_{1} u^{-\beta}, \quad u \rightarrow \infty, \quad \beta=1-\gamma>0
$$

If $U(r) \rightarrow \infty$ as $r \rightarrow 0$, then, roughly speaking, we obtain for $u \rightarrow \infty$

$$
\sigma^{(2)}(u) \approx \text { const } r_{0}^{2}(u), \quad U\left(r_{0}\right)=\frac{m u^{2}}{4},
$$

where $m$ is a mass of particle, $r_{0}$ is a minimal distance between two particles with given relative energy $T=m u^{2} / 4$. Assuming that

$$
U(r) \approx \frac{\alpha}{r^{s}}, \quad r \rightarrow 0
$$

we obtain

$$
r_{0} \approx\left(\frac{4 n}{m u^{2}}\right)^{s}, \quad \sigma^{(2)}(u) \approx \text { const } \mathrm{u}^{-4 / s}, \mathrm{u} \rightarrow \infty .
$$

Hence, the first condition in $(60)$ with $\gamma=1-4 / s>0$ is fulfilled for a wide class of potentials with asymptotic behaviour ( 72 ), where $s>4$. In the opposite case $s \leq 4$ the inequalities $(62)$ are valid.

If the potential $U(r)$ has a hard core, i.e. $U(r) \rightarrow \infty$ as $r \rightarrow d_{\text {min }}$, then particles with large relative speed $u \rightarrow \infty$ interact like hard spheres with diameter $d_{\text {min }}$. Therefore $\gamma=1 \mathrm{in}(60)$ for this case,

Thus we proved for a wide class of intermolecular potentials $U(r)$ (including power-like potentials (3) for $s>4$ without cut-off and potentials with compact support satisfying conditions (60)) that all moments of the solution to the Boltzmann equation (1) are uniformly bounded for all $t>0$. This result is mainly interesting for slowly decreasing (with $|v| \rightarrow \infty)$ initial conditions with finite number of moments at $t=0$.

However such initial conditions are not typical for applications of the Boltzmann equation. Usually we have a distribution function with the Maxwellian tail and need to estimate a possible growth of the tail. The above obtained estimates (61) are too rough for this problem. Therefore we shall construct some more precise estimates in Section 6 for a special case of hard spheres.

\section{Solutions with Maxwellian tails.}

We consider the Boltzmann equation (1) for hard spheres (2) with the initial condition (5i). Our goal in this Section is to prove the following theorem.

Theorem 3. If

$$
\int d \mathbf{v} f_{0}(\mathbf{v}) \ln f_{0}(\mathbf{v})<\infty, \int d \mathbf{v} f_{0}(\mathbf{v}) e^{\theta r^{2}}<\infty
$$


for a certain $\theta>0$, then there exists $0<\theta_{*} \leq \theta$, such that for all $t>0$

$$
F\left(\theta_{*}, t\right)=\int d \mathrm{v} f(\mathrm{v}, t) e^{\theta_{*} v^{2}}<\infty
$$

Proof. First we note that

$$
F(z)=\int d \mathbf{v} f(\mathbf{v}) e^{z y^{2}}=\sum_{n=0}^{\infty} \frac{z^{n}}{n !} m_{n}, \quad m_{n}=\int d \mathbf{v} f(\mathbf{v}) v^{2 n}
$$

Therefore $F(z)$ is an analytic function for $|z|<R$, where

$$
R^{-1}=\lim \sup \left(\frac{m_{n}}{n !}\right)^{1 / n}
$$

To prove the theorem it is sufficient to estimate moments $m_{n}(t)$ to the solution by inequality

$$
m_{n}(t)<Q(n) a^{n} n !, \quad[Q(n)]^{1 / n} \rightarrow 1
$$

for certain $a>0$, provided that (73)

$$
\limsup \left[\frac{m_{n}(0)}{n !}\right]^{1 / n}=a_{0}<\theta^{-1} \text {. }
$$

All necessary estimates are given below in Lemmas $6.1-6.4$ provided that all moments $m_{p}(t)$ of integer and half-integer orders $p=1,3 / 2,2, \ldots$ are continuously differentiable Cunctions of $l \in R^{+}$.

Lemma 6.1. Moments

$$
m_{p}(t)=\int d \mathrm{v} f(\mathrm{v}, t) v^{2 p}, \quad p=3 / 2,2,5 / 2, \ldots
$$

satisfy the following set of inequalities

$$
\begin{gathered}
\frac{1}{\sigma} \frac{d m_{n}}{d t} \leq \frac{2}{n+1} \sum_{k=1}^{n-1}\left(\begin{array}{l}
n \\
k
\end{array}\right) m_{k+1 / 2} m_{n-k}-q \frac{n-1}{n+1} m_{n}^{1+1 / 2 n}, n=1,2,3, \ldots, \\
\frac{1}{\sigma} \frac{d m_{n+1 / 2}}{d t} \leq \frac{4}{2 n+3}\left\{2 \sum_{k=1}^{n-1}\left(\begin{array}{l}
n \\
k
\end{array}\right) m_{k+1 / 2} m_{n-k}+m_{1 / 2} m_{n+1 / 2}+m_{1} m_{n}\right\} \\
-q \frac{2 n-1}{2 n+3} m_{n+1 / 2}^{1+1 /(2 n+1)}, q=q\left(1, E_{0}, H_{0}\right) / \sigma, \quad n=1,2,3, \ldots ;
\end{gathered}
$$

where $q\left(1, E_{0}, H_{0}\right)$ is defined in $(64)$.

Proof. The above obtained inequality (12), (16) for hard spheres results in

$$
\begin{gathered}
\frac{1}{\sigma} \frac{d m_{p}}{d t} \leq \frac{1}{p+1} \int d \mathbf{v} d \mathbf{w} f(\mathbf{v}, t) f(\mathbf{w}, t)|\mathbf{v}-\mathbf{w}| \times \\
\times\left\{\left[\left(v^{2}+w^{2}\right)^{2 p}-v^{2 p}-w^{2 p}\right]-\frac{1}{2}(p-1)\left(v^{2 p}+w^{2 p}\right)\right\}=S_{p}^{+}-S_{p}^{-}, \quad p \geq 1 .
\end{gathered}
$$


Using Carleman's estimate (64) with $\gamma=1$ for the collision frequency and Höler's inequality (66) we obtain

$$
s_{p} \geq q \frac{p-1}{p+1} m_{p+1 / 2} \geq q \frac{p-1}{p+1} m_{p}^{1+1 / 2 p}
$$

that is just the negative term in (77)-(78). To get the positive term in (77) it is sufficient to use a trivial estimate $|\mathbf{v}-\mathbf{w}| \leq v+w$ in (79) for $p=n$. The positive term in (78) follows from incqualitios

$$
\begin{gathered}
|\mathrm{v}-\mathrm{w}|\left[\left(v^{2}+w^{2}\right)^{n+1 / 2}-v^{2 n+1}-w^{2 n+1}\right] \leq \\
\leq(v+w)\left\{\left(v^{2}+w^{2}\right)^{1 / 2} \sum_{k=1}^{n-1}\left(\begin{array}{l}
n \\
k
\end{array}\right) v^{2 k} w^{2(n-k)}+v^{2 n}\left[\left(v^{2}+w^{2}\right)^{1 / 2}-v\right]+w^{2 n}\left[\left(v^{2}+w^{2}\right)^{1 / 2}-w\right]\right\} \leq \\
\leq 2\left(v^{2}+w^{2}\right) \sum_{k=1}^{n-1}\left(\begin{array}{l}
n \\
k
\end{array}\right) v^{2 k} w^{2(n-k)}+(v+w)\left[v^{2 n} w+w^{2 n} v\right] .
\end{gathered}
$$

It completes the proof.

We assume that $m_{0}(t)=1, m_{1}(t)=E_{0}=$ const. The first $(n=1)$ equation $(78)$ includes $m_{1 / 2}(t)$. To eliminate this quantity one can use an obvious inequality

$$
m_{1 / 2} \leq \frac{1}{2}\left[\alpha m_{0}+\frac{m_{2}}{\alpha}\right]
$$

with appropriate $\alpha>0$.

Let us change variables in $(77),(78)$ putting

$$
m_{p}(t)=A \Gamma(p+1 / 2) a^{p} z_{p}(\tau), \quad \tau=A \sigma \sqrt{a} t, \quad p=1,3 / 2,2, \ldots
$$

with certain positive constants $A$ and $a$.

Moreover we estimate $m_{1 / 2}$ by the inequality (81) with $\alpha=a^{1 / 2}$, then

$$
m_{1 / 2}(t) \leq \frac{1}{2} a^{1 / 2}\left[1+A \Gamma(3 / 2) z_{1}\right], \quad A \Gamma(3 / 2) z_{1}=\frac{E_{0}}{a} .
$$

Thus we obtain the following set of inequalities for $z_{p}(\tau)$ :

$$
\begin{aligned}
\frac{d z_{n}}{d \tau} \leq & \frac{2}{n+1} \sum_{k=1}^{n-1}\left(\begin{array}{l}
n \\
k
\end{array}\right) \frac{\Gamma(k+1) \Gamma(n-k+1 / 2)}{\Gamma(n+1 / 2)} z_{k+\frac{1}{2}} z_{n-k}- \\
& \left.-q \frac{n-1}{n+1} A^{-(1-1 / 2 n)} \Gamma(n+1 / 2)\right]^{1 / 2 n}\left(z_{n}\right)^{1+1 / 2 n}, \\
\frac{d z_{n+\frac{1}{2}}}{d \tau} \leq & \frac{4}{2 n+3}\left[2 \sum_{k=1}^{n-1}\left(\begin{array}{l}
n \\
k
\end{array}\right) \frac{\Gamma(k+3 / 2) \Gamma(n-k+1 / 2)}{\Gamma(n+1)} z_{k+1} z_{n-k}\right. \\
+ & \frac{\Gamma(3 / 2) \Gamma(n+1 / 2)}{\Gamma(n+1)} z_{1} z_{n}+\frac{1}{2}\left(A^{-1}+\Gamma(3 / 2) z_{1}\right) z_{n+\frac{1}{2}}
\end{aligned}
$$




$$
-q \frac{2 n-1}{2 n+3}[\Gamma(n+1)]^{\frac{1}{2 n+1}} A^{-\left(1-\frac{1}{2 n+1}\right)} z_{n+1 / 2}^{1+\frac{1}{2 n+1}}, \quad n=2, \ldots
$$

The inequalities can be simplified by using some estimates for gamma-function.

Lemma 6.2. It follows from (84), (85) that

$$
\begin{aligned}
& \quad \frac{d z_{n}}{d \tau} \leq 4 \max _{1 \leq k \leq n-1}\left(z_{k+\frac{1}{2}} z_{n-k}\right)-\frac{q}{3} A^{-\left(1-\frac{1}{2 n}\right)} z_{n}^{1+\frac{1}{2 n}}, \\
& \frac{d z_{n+1 / 2}}{d \tau} \leq 2 \pi\left(1-\delta_{n-1}\right) \max _{1 \leq k \leq n-1}\left(z_{k+1} z_{n-k}\right)+\frac{\pi}{5} z_{1} z_{n}+ \\
& +\frac{1}{2 A}\left(1+\frac{E_{0}}{a}\right) z_{n+1 / 2}-\frac{q}{5} A^{-\left(1-\frac{1}{2 n+1}\right)} z_{n+\frac{1}{2}}^{1+\frac{1}{2 n+1}}, \quad n=1, \ldots
\end{aligned}
$$

Proof. We note that [9]

$$
\Gamma(z+1)=z \Gamma(z), \quad \frac{\Gamma(x) \Gamma(y)}{\Gamma(x+y)}=B(x, y)=\int_{0}^{1} d s s^{x-1}(1-s)^{y-1}
$$

Therefore

$$
\begin{gathered}
\frac{2}{n+1} \sum_{k=1}^{n-1}\left(\begin{array}{l}
n \\
k
\end{array}\right) \frac{\Gamma(k+1) \Gamma(n-k+1 / 2)}{\Gamma(n+1 / 2)}= \\
=\frac{2 \Gamma(n+3 / 2)}{(n+1) \Gamma(n+1 / 2)} \sum_{k=1}^{n-1}\left(\begin{array}{l}
n \\
k
\end{array}\right) B(k+1, n-k+1 / 2)= \\
=\frac{2 n+1}{n+1} \int_{0}^{1} d s s^{-1 / 2}\left[1-s^{n}-(1-s)^{n}\right] \leq 4, \\
\frac{8}{2 n+3} \sum_{k=1}^{n}\left(\begin{array}{l}
n \\
k
\end{array}\right) \frac{\Gamma(k+3 / 2) \Gamma(n-k+1 / 2)}{\Gamma(n+1)}= \\
=\frac{8 \Gamma(n+2)}{(2 n+3) \Gamma(n+1)} \sum_{k=1}^{n-1}\left(\begin{array}{l}
n \\
k
\end{array}\right) B(k+3 / 2, n-k+1 / 2)- \\
=\frac{8(n+1)}{2 n+3} \int_{0}^{1} d s s^{1 / 2}(1-s)^{-1 / 2}\left[1-s^{n}-(1-s)^{n}\right] \leq 4 B(3 / 2,1 / 2)=2 \pi .
\end{gathered}
$$

It gives the above estimates (86), (87) for sums in (84), (85). To complete the proof we estimate other terms in (84), (85) by some obvious inequalities: $\Gamma(p+1 / 2) \geq 1$ for $p \geq 3 / 2,(n-1) /(n+1) \geq 1 / 3$ for $n \geq 2$ and

$$
\frac{4 \Gamma(3 / 2) \Gamma(n+1 / 2)}{(2 n+3) \Gamma(n+1)} \leq \frac{\pi}{2 n+3} \leq \frac{\pi}{5}, \quad \frac{2 n-1}{2 n+3} \geq \frac{1}{5}
$$

for $n \geq 1$. Lemma 6.2 is proved. 
We note that inequalities (86), (87) are valid for any positive $A$ and $a$. The next step is to choose $A$ and $a$ in such a way that the inequality $z p(t) \leq 1, p=1,3 / 2,2, \ldots$, holds for all $t>0$ if it is filfilled for $t=0$. First we restrict a by the condition.

$$
a \geq E_{0} \max (2 A-1, A \Gamma(3 / 2)),
$$

then

$$
z_{1}=\frac{E_{0}}{A a \Gamma(3 / 2)} \leq 1, \quad \frac{1}{2 A}\left(1+\frac{E_{0}}{a}\right) \leq 1 .
$$

Let us now combine (86) and (87) and consider simplified inequalities

$$
\begin{aligned}
& \frac{d z_{p}}{d T} \leq F_{p}\left(z_{1}, \ldots, z_{p-1 / 2}\right)+z_{p}-\lambda A^{-\left(1-\alpha_{p}\right)} z_{p}^{1+\alpha_{p}} \\
& \lambda=q / 3 \quad \alpha_{p}=1 / 2 p, \quad F_{p \geq 2}=8 \max _{1 \leq k \leq[p]}\left(\alpha_{k} z_{p} k_{p+\frac{1}{2}}\right), \\
& F_{3 / 2}=\pi / 5, \quad p=3 / 2,2,5 / 2, \ldots \text {. }
\end{aligned}
$$

A solution of differential inequalities $(90)$ can be estimated by the following lemma.

Lemma 6.3. If $x(t)$ satysfies the inequality

$$
\frac{d x}{d t} \leq B+x-C x^{1+1 / 2}
$$

with certain positive constants $B, C, q$, then

$$
x(t) \leq \max \left[x(0), x_{*}\right]
$$

where $a_{*}$ is a unique positive root of the algebraic equation

$$
B+x_{*}=C x_{*}^{1+1 / q}
$$

moreover

$$
x(t) \leq x_{*}\left[1-c^{-3 t}\right]^{-q}, \quad \beta=q^{x_{*}^{1 / q}}
$$

Remark. In this Section we need only the inequality (92), The second inequality (94) is necessary to complete the proof of Theorem 2 in Section 5.

Proof. Changing variables in (91) we obtain

$$
x(t)=[y(t / q)]^{-q}, \quad \frac{d y}{d t}+y\left[1+B y^{q}\right] \geq C
$$

We note that there exists a unique number $y_{*}>0$, such that

$$
y_{*}\left[1+B y_{*}^{q}\right]=C
$$

If $y<y_{*}$, then $y_{t}>0$. Therefore $y(t) \geq y(0)$ for all $t \geq 0$ if $y(0)<y_{*}$. Moreover $y(t)<y_{*}$ in this case and

$$
y(t) \geq O \int_{0}^{t} d t_{1} \exp \left[-\int_{t_{1}}^{t} d \tau\left\{1+B y^{\prime}(\tau)\right\}\right] \geq
$$




$$
\geq C \int_{0}^{t} d t_{1} \exp \left[-\left(1+B y_{*}^{q}\right)\left(t-t_{1}\right)\right]=y_{*}\left[1-\exp \left(-\frac{c t}{y_{*}}\right)\right]
$$

If $y(0) \geq y_{*}$, then for a similar reason $y(t) \geq y_{*}$ for all $t>0$. Therefore the formula (96) is correct also for this case. To complete the proof we need only to re-formulate the last inequalities in terms of $x(t)(95)$.

Thus, to obtain a desirable estimate $z(\tau) \leq 1$ for a solution of $(90)$ it is sufficient to satisty the following conditions $(p=3 / 2,2, \ldots)$ :

$$
0 \leq z_{p} \leq 1, \quad 0 \leq z_{p}^{*} \leq 1
$$

where $z_{p}^{*}$ are defined by equations (see $(90)$ )

$$
F_{p}\left(z_{1}^{*}, \ldots, z_{p-1 / 2}^{*}\right)+z_{p}^{*}=\lambda A^{-(1-1 / 2 p)}\left(z_{p}^{*}\right)^{1+1 / 2 p}
$$

We note that $A>0$ is still a free parameter. To satisty the second inequality it is sufficient to choose $A$ in such a way that

$$
\lambda A^{-(1-1 / 2 p)} \geq 9 \geq 1+\max F_{p}, \quad p=3 / 2,2, \ldots
$$

since $F_{3 / 2}=\pi / 5$ and $F_{p} \leq 8$ for $p \geq 2$ if $\max \left(z_{1}^{*}, \ldots, z_{p-1 / 2}^{*}\right) \leq 1$. Thus we obtain a condition $A \leq \min \left[1,(\lambda / 9)^{3 / 2}\right], \lambda=q / 3$ which garantees that $z_{p}^{*} \leq 1$ for all $p \geq 3 / 2$. However $q \leq 1$ in $(64)$ since

$$
\lim _{|\mathbf{v}| \rightarrow \infty} \frac{1}{|\mathbf{v}|} \int d \mathbf{w} f(\mathbf{w})|\mathbf{v}-\mathbf{w}|=m_{0}=1
$$

Therefore to fulfil the second condition (97) it is sufficient to put

$$
A=(q / 27)^{3 / 2}
$$

The result can be formulated in the following way.

Lemma 6.4. Let $m_{0}=1, m_{1}=E_{0}, m_{p}(t)$ for $p=3 / 2,2, \ldots$ satisfy inequalities $(77)$, (78) with $q \leq 1$ for $t>0$ and initial inequalities

$$
m_{p}(0) \leq(q / 27)^{3 / 2} a^{p}, \quad p=1,3 / 2,2, \ldots
$$

for certain $a>0$. Then the inequalities

$$
m_{p}(t) \leq(q / 27)^{3 / 2} a^{p}, \quad p=1,3 / 2,2, \ldots
$$

hold for all $t>0$.

Proof. We reduce (77), (78) to (86), (87) by Lemma 6.2. Then we apply Lemma $6.3(92)$ to the first inequality for $p=3 / 2$. The constant $A$ satisfies equality (98). The condition (99) for $p=1$ and the estimate $A=(q / 27)^{3 / 2}<1 / 2$ guarantee that the constant $a$ satisfies (89). Therefore (100) is correct for $p=3 / 2$. By induction on $p=2, \ldots$ we repeat the same arguments and complete the proof. 
Theorem 3 follows directly from Lemmas 6.1 and 6.4 since the condition (73) guarantees that there exists $a>0$ such that inequalities (99) are satisfied. Then (100) shows that the integral (74) with $\theta_{*}=a^{-1}$ converges for all $t>0$.

Remark. The function

$$
\tau(t)=\frac{1}{2}\left[\sup \theta_{*}\right\}^{-1}
$$

where supremum is taken over all $\theta_{*}>0$ satisfying $(74)$ for any fixed $t>0$, is called the tail temperature. Its properties for Maxwell molecules were studied in detail in [1]. It is also known [11] that $\tau(t)$ is a monotonically non-decreasing function of $t$ for hard spheres and potentials with compact support.

\section{Generalizations.}

The result of Section 6 can be generalized in different ways. First we prove a simple inequality for the collision frequency and generalize Theorem 3 to a wider class of initial conditions.

Lemma 7.1. If $m_{0}(t)=1$, then the collision frequency

$$
\nu(\mathbf{v}, t)=\sigma \int d \mathbf{w} f(\mathbf{w}, t)|| \mathbf{v}-\mathbf{w} \mid
$$

in the Boltzmann equation (1) for hard spheres satisfies the inequality

$$
\nu(\mathrm{v}, t) \geq 7^{-1 / 2} \nu(\mathrm{v}, 0)
$$

Proof. We note that $\psi(z)=-z^{1 / 2}$ is obviously a convex function. Thercfore wc obtain by the inequality (12) with $A_{+}\left[-z^{1 / 2}\right]$ for hard spheres (16) the following lower estimate for $m_{1 / 2}(t)$ :

$$
\frac{1}{\sigma} \frac{d m_{1 / 2}}{d t} \geq \int d \mathbf{v} d \mathbf{w} f(\mathbf{v}, t) f(\mathbf{w}, t)|\mathbf{v}-\mathbf{w}|\left\{\frac{2}{3} \sqrt{v^{2}+w^{2}}-\frac{1}{2}(v+w)\right\}
$$

Using obvious inequalities

$$
|\mathrm{v}-\mathrm{w}| \sqrt{v^{2}+w^{2}} \geq(v-w)^{2}, \quad|\mathrm{v}-\mathbf{w}|(v+w) \leq(v+w)^{2}
$$

we get a simple expression

$$
\frac{3 d m_{1 / 2}}{d t} \geq m_{0} m_{1}-7 m_{1 / 2}^{2}, \quad m_{0}=1, \quad m_{1}=E_{0}
$$
that

Noting then $\left(m_{1 / 2}\right)_{i}$ is always non-negative for $m_{1 / 2}^{2} \leq E_{0} / T$ one can easily conclude

$$
m_{1 / 2}(t) \geq \min \left\{m_{1 / 2}(0),(E / T)^{1 / 2}\right\} \text {. }
$$

Moreover $E \geq m_{1 / 2}^{2}$ (a special case of $(66)$ ), therelore we obtan an estimate

$$
m_{1 / 2}(t) \geq \frac{1}{\sqrt{7}} m_{1 / 2}(0)
$$


The collision frequency $\nu(\mathbf{v}, t)$ in the Boltzmann equation (1) for hard spheres reads

$$
\nu(\mathbf{v}, t)=\sigma \int d \mathbf{w} f(\mathbf{w}+\mathbf{v}, t)|\mathbf{w}|
$$

We note that for any fixed $v_{0} \in R^{3}$ the function $f\left(v+v_{0}, t\right)$ satisfies the same Boltzmann equation (1) with initial condition $f_{\mid t=0}=f_{0}\left(v+v_{0}\right)$. Therefore the inequality (102) follows directly from (103).

Corollary. The entropy assumption (73) in Theorem 3 can be replaced by a weaker assumption

$$
\int d \mathbf{w} f_{0}(\mathbf{w})|\mathbf{v}-\mathbf{w}| \geq c_{0}|\mathbf{v}|
$$

then all above obtained estimates are valid for $q=7^{-1 / 2} c_{0}$ in formulas $(77)-(100)$.

It is clear that estimates similar to (102) can be proved for a wide class of potentials with compact support by using the inequality (56). Unfortunatcly this inequality is not enough for a similar proof of Theorem 3 in the general case. However a weaker result can be easily obtained.

Theorem 4. Let $f(\mathrm{v}, t)$ be a solution of the Boltzmann equation (1) for the potential with compact support. If the initial condition $f_{0}(\mathrm{v})$ satisfies the inequality

$$
\int d \mathbf{v} f_{0}(\mathbf{v}) e^{\theta v^{2}}<\infty
$$

for a certain $\theta>0$, then there exist $0<\theta_{*} \leq \theta$ and $\alpha>0$, such that for all $t>0$

$$
\int d \mathbf{v} f(\mathbf{v}, t) \exp \left[\theta_{*} e^{-\alpha t} v^{2}\right]<\infty
$$

Proof. The proof is based on the simplest estimate of $A\left[z^{p}\right]$ in (6). Noting that

$$
v^{2 p}+w^{2 p} \leq\left(v^{2}+w^{2}\right)^{p}, \quad v^{2}+w^{2}=v^{2}+w^{2}
$$

for $p \geq 1$ and therefore

$$
v^{2 p}+w^{2 p} \leq\left(v^{2}+w^{2}\right)^{p}
$$

we immediately obtain from (5)-(6) the inequality

$$
\frac{d m_{p}}{d t} \leq \frac{\sigma}{2} \int d \mathbf{v} d \mathbf{w} f(\mathbf{v}, t) f(\mathbf{w}, t) u\left[\left(v^{2}+w^{2}\right)^{p}-v^{2 p}-w^{2 p}\right], \quad p \geq 1
$$

where $\sigma=$ const (46) for potentials with compact support.

Then we repeat first steps of the proof of Theorem 3 almost without any changes. The only difference is that we substitute the estimate (12), (16) for hard spheres by the simplest inequality (105). Following the proof of Lemma 6.1 we obtain in general case not the inequalities (77), (78), but weaker estimates

$$
\begin{gathered}
\frac{1}{\sigma} \frac{d m_{n}}{d t} \leq \sum_{k=1}^{n-1}\left(\begin{array}{l}
n \\
k
\end{array}\right) m_{k+\frac{1}{2}} m_{n-k}, \\
\frac{1}{\sigma} \frac{d m_{n+1 / 2}}{d t} \leq 2 \sum_{k=1}^{n-1}\left(\begin{array}{l}
n \\
k
\end{array}\right) m_{k+1} m_{n-k}+m_{\frac{1}{2} m_{n+\frac{1}{2}}}+m_{1} m_{n}
\end{gathered}
$$


Then we use the same substitution (82) with $A=1$ and repeat the proof of Lemma 6.2 . It results in inequalities

$$
\begin{gathered}
\frac{d z_{n}}{d \tau} \leq 2(n+1) \max _{1 \leq k \leq n}\left(z_{k+1} z_{n-k}\right), \\
\frac{d z_{n+1 / 2}}{d \tau} \leq \frac{\pi}{2}(2 n+3)\left(1-\delta_{n 1}\right) \max _{1 \leq k \leq n}\left(z_{k+1} z_{n-k}\right)+ \\
+\frac{\pi}{5} z_{1} z_{n}+\frac{1}{2}\left(1+\frac{E_{0}}{a}\right) z_{n+1 / 2}, n=1,2, \ldots,
\end{gathered}
$$

which replace inequalities $(86),(87)$. Then we again use an assumption (88) (it is sufficient to choose $a<\sqrt{\pi} / 2 E_{0}$ ) and replace $(90)$ by a simpler inequality

$$
\frac{d z_{p}}{d \tau} \leq 4(p+1) \max _{1 \leq k \leq[p]}\left(z_{k} z_{n-k+1 / 2}\right)+z_{p}, \quad p=2,5 / 2, \ldots, \quad \frac{d z_{3 / 2}}{d \tau} \leq \frac{\pi}{4}+z_{3 / 2}
$$

We assume that the constant $a$ in $(82)($ with $A=1)$ is chosen in such a way that

$$
m_{p}(0) \leq \Gamma(p+1 / 2) a^{p}, \quad p=1,3 / 2, \ldots
$$

then $z_{p}(0) \leq 1$ in $(106)$. A standard estimate for $z_{3 / 2}(\tau)(106)$ results in

$$
z_{3 / 2}(\tau)<e^{\tau}\left[z_{3 / 2}(0)+\frac{\pi}{4}\left(1-e^{-t}\right)\right]<\exp \left[\left(1+\frac{\pi}{4}\right) \tau\right] .
$$

For $p \geq 2$ wo put

$$
z_{p}(\tau)=y_{p}(\tau) \exp \left[\beta\left(p-\frac{1}{2}\right) \tau\right], \beta \geq 1+\frac{\pi}{4}
$$

then

$$
\frac{d y_{p}}{d \tau} \leq 4(p+1) \max _{1 \leq k \leq[p]}\left(y_{k} y_{n-k+1 / 2}\right)-\left[\beta\left(p-\frac{1}{2}\right)-1\right] y_{p}
$$

We note that

$$
y_{p}(0) \leq 1, \quad y_{1}(\tau) \leq 1, \quad y_{3 / 2}(\tau) \leq 1
$$

and choose $\beta=9$. Then $4(p+1)<\beta(p-1 / 2)-1$ for all $p \geq 2$. Therefore

$$
y_{p}(\tau) \leq y_{p}(0) \leq 1, \quad p=1,3 / 2, \ldots
$$

Hence, inequalities

$$
m_{p}(t)=\Gamma(p+1 / 2) a^{p} z_{p}(T) \leq \Gamma(p+1 / 2) a^{p} e^{\alpha(p-1 / 2) t}, \quad \alpha=9 \sigma \sqrt{a}, \quad p=1,3 / 2, \ldots .
$$

are valid for any $t>0$ if they are valid at $t=0$. To complete the proof we use the formula (75) and conclude that inequality (105) is fulfilled with $\alpha=9 \sigma \sqrt{a}$ and $\theta_{*}=a^{-1}$, where $a$ is chosen in such a way that

$$
m_{p}(0) \leq \Gamma(p+1 / 2) a^{p}, \quad p=1,3 / 2 \ldots
$$


Remark 1. Theorem 4 can be considered as an a priori estimate which shows that there exists a global solution of the Cauchy problem for spatially homogeneous Boltzmann equation (potentials with compact support) in the class of distribution functions with Maxwellian tails. This class is defined by the only condition: there exists such number $\theta>0$ that

$$
\int d \mathbf{v} f(\mathbf{v}) \exp \left(\theta v^{2}\right)<\infty
$$

Remark 2. It is clear that the results of Sections $6-7$ can be also formulated in the following way : if there exist positive constants $A$ and $B$ such that $m_{p}(0)$ are less than corresponding moments of the Maxwellian $A \exp \left(-B v^{2}\right)$, then there exist a constant $A_{1}>0$ and a function $B_{1}(t)>0$, such that $m_{p}(t)$ are bounded by corresponding moments of the Maxwellian $A_{1} \exp \left[-B_{1}(t) v^{2}\right]$ for any $t>0$. Moreover, $B_{1}(t)=B_{1}(0)=$ const for hard spheres, and $B_{1}(t)=B_{1}(0) \exp (-\alpha t)$ for a general potential with compact support. Constants $B_{1}(0)$ and $\alpha$ depend only on the total cross section $\sigma$ and initial condition $f_{0}(\mathrm{v})$.

\section{Acknowledgment.}

I am grateful to C.Cercignani for fruitful discussions about entropy inequalities which were a starting point for this paper. A material of Sections 6-7 was initiated by a discussion with L. Arkeryd, I thank him. It is my pleasure to thank H.Neunzert for discussions and his kind hospitality in Kaiserslautern, where a part of this paper was written. The support from Russian Foundation for Basic Research (grant No.96-01-00084) is also acknowledged.

\section{References}

[1] A.V.Bobylev. The theory of the nonlinear spatially uniform Boltzmann equation for Maxwell molecules. Sov. Sci. Rev. C, Vol. 7 (1988), pp. 111-233.

[2] B.Wennberg. Entropy dissipation and moment production for the Bollzmann equation. Preprint No 1995-38 (1995), Gotteborg University.

[3] L.Desvillettes. Some applications of the method of moments for the homogeneous Boltzmann and Kac equations, Arch. Rational Mech. Anal., Vol. 123 (1993), pp.387400.

[4] A.Ya.Povzner. On the Boltzmann equation in kinetic theory of gases. Mat. Sbornik (1962) Vol. 58(100), No 1, pp. 65-86 (In Russian).

[5] C.Cercignani, R.Illner, M.Pulvirenti. The Mathematical Theory of Dilute Gases, Springer, New York (1994).

[6] T.Elmroth. Global boundedness of moments of solutions of the Boltzmann equation for forces of infinite range. Arch. Rational Mech. Anal., Vol. 82 (1983), pp. 1-12.

[7] W.Feller. An Introduction to Probability I'heory and Its Applications. Vol.II, John Wiley and Sons, New York, 1971.

[8] K.M.Case, P.Zweifel, Linear Transport Theory, Reading, Addison-Wesley, 1967. 
[9] I.S.Gradsteyn, I.M.Ryzhik. Tables of Integrals, Series and Products, Academic Press, San Diego, 1980.

[10] L.Arkeryd. L estimates for the space homogeneous Boltzmann equation. J. Stat. Phys., Vol.31 (1982), pp.347-361.

[11] A.V.Bobylev, G. Toscani. Generalization of the Boltzmann H-theorem for Maxwell gas. J. Math. Phys. Vol. 33 (1992), pp.443-476. 